\title{
Evaluation of Bone Metabolism in Critically Ill Patients Using CTx and PINP
}

\author{
Alexandra Gavala, ${ }^{1}$ Konstantinos Makris, ${ }^{2}$ Anna Korompeli, ${ }^{1}$ and Pavlos Myrianthefs ${ }^{1}$ \\ ${ }^{1}$ School of Health Sciences, Department of Nursing, "Agioi Anargyroi" General Hospital, National and Kapodistrian \\ University of Athens, Noufaron \& Timiou Stavrou, 14564 Kaliftaki, Nea Kifissia, Athens, Greece \\ ${ }^{2}$ Clinical Biochemistry Department, KAT General Hospital, Kifissia, Athens, Greece \\ Correspondence should be addressed to Pavlos Myrianthefs; pmiriant@nurs.uoa.gr
}

Received 9 August 2016; Revised 11 October 2016; Accepted 15 November 2016

Academic Editor: Nikolaos K. Kanakaris

Copyright (c) 2016 Alexandra Gavala et al. This is an open access article distributed under the Creative Commons Attribution License, which permits unrestricted use, distribution, and reproduction in any medium, provided the original work is properly cited.

Background. Prolonged immobilization, nutritional and vitamin D deficiency, and specific drug administration may lead to significant bone resorption. Methods and Patients. We prospectively evaluated critically ill patients admitted to the ICU for at least 10 days. Demographics, APACHE II, SOFA scores, length of stay (LOS), and drug administration were recorded. Blood collections were performed at baseline and on a weekly basis for five consecutive weeks. Serum levels of PINP, $\beta$-CTx, iPTH, and $25(\mathrm{OH})$ vitamin D were measured at each time-point. Results. We enrolled 28 patients of mean age $67.4 \pm 2.3$ years, mean APACHE II $22.2 \pm 0.9$, SOFA $10.1 \pm 0.6$, and LOS $31.6 \pm 5.7$ days. Nineteen patients were receiving low molecular weight heparin, 17 norepinephrine and low dose hydrocortisone, 18 transfusions, and 3 phenytoin. $25(\mathrm{OH})$ vitamin $\mathrm{D}$ serum levels were very low in all patients at all time-points; iPTH serum levels were increased at baseline tending to normalize on 5 th week; $\beta$-CTx serum levels were significantly increased compared to baseline on 2nd week (peak values), whereas PINP levels were increased significantly after the 4th week. Conclusions. Our data show that critically ill patients had a pattern of hypovitaminosis D, increased iPTH, hypocalcaemia, and BTMs compatible with altered bone metabolism.

\section{Introduction}

Critical illness may accelerate or lead to altered bone turnover due to prolonged immobilization, nutritional defects, inflammation, vitamin $\mathrm{D}$ deficiency, exposure to medications that affect bone and calcium metabolism, and endocrine dysfunction. These factors, separately or in combination, may contribute to increased bone resorption and decreased bone formation [1]. This accelerated bone turnover may contribute to the burden of morbidity and mortality observed in survivors of intensive care $[2,3]$. In patients with prolonged critical illness, circulating biomarkers of bone resorption are substantially elevated whereas markers of bone formation are low [4]. Also, the measurement of bone turnover markers (BTMs) reveals markedly enhanced osteoclastic bone resorption that is uncoupled from osteoblastic bone formation [5].

Such an imbalance of BTMs may predispose critically ill patients to impaired fracture healing, osteoporosis, and increased risk of new fractures during intensive care unit (ICU) stay or rehabilitation. A recent retrospective casecohort study revealed a significant increase in fracture risk in survivors of critical illness, implying a clinically relevant impact of the reported alterations in bone biomarkers [6].

Although data are limited until now a positive association (moderate evidence) between critical illness requiring intensive care admission and increased bone turnover exists according to a recent systematic review [1]. However, the extent, risk factors, and the nature of the relationship between critical illness and bone turnover are not yet clearly understood [1].

Also, studies have employed different methodologies and recruited heterogenous populations (differences in ages, gender, and population sizes) with varied duration of followup [1]. Most importantly they examined different serum or urinary markers of bone turnover which are not well documented and in variable timeframes. 
Currently, the measurements of serum levels of Ctelopeptide fragments of collagen type I al chains $(\beta-\mathrm{CTx})$ and N-terminal propeptide of type I procollagen (PINP) are recommended as markers of bone resorption and bone formation markers, respectively, correlated with corresponding histomorphometric parameters of bone formation and resorption [7]. However this recommendation is applicable to assess progression of osteoporosis, fracture risk, and treatment response.

Serum $\beta$-CTx is a bone resorption marker of choice in view that the assay is well characterized, measures an 8amino-acid peptide, has been evaluated in many studies, and is widely available as an automated immunoassay or manual ELISA, and the biological and analytical variability is well documented [7]. Serum PINP is the reference standard for bone formation, because most PINP probably is produced during bone formation, the assay is widely available as an automated immunoassay or manual radioimmunoassay, and the biological and analytical variability is well known [7]. These two markers have been studied extensively in patients with osteoporosis but were only occasionally evaluated in critically ill patients and especially $\beta$-CTx in only two studies and PINP in another $[4,8,9]$. Therefore more data are needed to establish their validity in this heterogeneous group of patients.

The aim of the study was to measure the changes of the levels of two biochemical markers of bone metabolism PINP and $\beta$-CTx along with total 25-hydroxyvitamin D $(25-\mathrm{OH}$ vitamin $\mathrm{D}$ ), and intact parathormone (iPTH) serum levels in critically ill patients during their ICU stay until discharge.

\section{Patients and Methods}

2.1. Study Design. In this prospective observational study we enrolled adult patients admitted to the ICU requiring mechanical ventilation expected to have at least 10 days of ICU stay and until discharge. Exclusion criteria included pediatric patients, pregnancy, renal, metabolic, and liver disease, metabolic and neurological disease, known osteoporosis, and prior medications affecting bone metabolism.

We prospectively collected demographics, admission diagnosis, common laboratory data routinely monitored for critically ill patients, APACHE II score, SOFA score, length of stay (LOS), drug administered including that affecting bone turnover such as low molecular weight heparin (LMWH), phenytoin, low dose steroids for septic shock (50 $\mathrm{mg} \times 4$ i.v.), and transfusions.

Fourteen healthy volunteers matched for age and sex (7 males and 7 females) of mean age (yrs) $65.2 \pm 6.4$ (median; 59, range 52-75 years) served as controls for $\beta$-CTx, PINP, iPTH, and total 25-hydroxyvitamin D serum levels for comparisons. These were historical samples randomly selected from a pool of data kept in the biochemistry department by selecting from a list one individual every five individuals until reaching 7 individuals.

2.2. Biochemical Measurements. Peripheral blood was collected at each time-point, between 08.00 and 10.00 A.M placed in tubes containing EDTA, centrifuged, and then stored in polypropylene tubes at $-20^{\circ} \mathrm{C}$ until measurements. Time-points were baseline (within $24 \mathrm{~h}$ of ICU admission) and on a weekly basis until ICU discharge or death. Blood collection was performed at least 5 days far from death. Serum levels of iPTH, 25-OH vitamin D, $\beta$-CTx, and PINP were determined with an electrochemiluminescence immunoassay (ECLIA) on Cobas e411 (Roche, Manheim, Germany). The sensitivity of this assay for $\mathrm{iPTH}$ is $1.2 \mathrm{pg} / \mathrm{mL}$, and the intra- and interassay CVs are 4 and $4.3 \%$, respectively. The sensitivity of this assay for 25-hydroxyvitamin $\mathrm{D}$ is $<5.0 \mathrm{ng} / \mathrm{mL}$, and the intra- and interassay CVs are 4.4 and $4.7 \%$, respectively. The total precision $(\mathrm{CVt})$ of these assays for $\beta$-CTX and PINP is $<3.5 \%,<1.8 \%$ and $<4.5 \%$, respectively.

2.3. Ethics. The study protocol was approved by the Scientific and Ethics Committees of the Hospital and waived the need for informed consent due to the nature of the study design. As patient care in the ICUs includes routine blood sampling for laboratory tests, no written informed consent was required from the patients.

2.4. Statistical Analysis. Qualitative characteristics are presented as numbers and percentages. Numerical data are expressed in mean \pm SEM or SD or percentages. BTMs values on figures and the table are presented as median $( \pm I Q R)$.

Comparisons were performed using nonparametric test Kruskal-Wallis test with Dunn's posttest analysis suitable for multiple comparisons (between all pairs). Statistical significance was considered at the level of $p<0.05$.

\section{Results}

3.1. Patient Demographics. We enrolled 28 patients (16 males) fulfilling the inclusion criteria. Patient demographics, medications, and laboratory measurements on admission are shown in Table 1. All patients had a pre-ICU stay in hospital wards or emergency department ranging from 1 to 3 days. All patients received enteral nutrition and supplemental micronutrients (Addamel ${ }^{\mathrm{TM}} \mathrm{N} 10 \mathrm{~mL}$, Fresenius Kabi USA, LLC) and vitamins (Cernevit ${ }^{\mathrm{TM}}$, Baxter Healthcare Ltd., A, B complex, C, D, E, and folic acid) in enriched intravenous fluids as medically indicated by physicians in charge. None of the patients experienced altered renal function tests (Table 1) that could influence bone turnover markers clearance from the circulation and thus their serum levels.

\subsection{Markers of Bone Turnover}

3.2.1. Control Group Values. BTMs for controls are shown in Table 2 together with consecutive BTM values for the patients.

3.2.2. Bone Resorption. Serum $\beta$-CTX levels were normal (Table 2, Figure 1(a)) in $55.2 \%$ of the patients on admission (baseline). Serum levels were increased in $82.1 \%, 84.2 \%, 60 \%$, $50 \%$, and $57.1 \%$ of the patients on 1 st, $2 \mathrm{nd}, 3 \mathrm{rd}, 4 \mathrm{th}$, and 5 th week, respectively. On the 2 nd week (peak levels) $\beta$ CTX serum levels were 2 -fold times greater compared to 
TABLE 1: Demographics, admission diagnosis, and characteristics of the patients $(n=28)$.

\begin{tabular}{|c|c|}
\hline Variable & Values \\
\hline Age, (range) yrs & $67,4 \pm 2,1(45-88)$ \\
\hline Male, $n, \%$ & $16,(57,1)$ \\
\hline \multicolumn{2}{|l|}{ Admission diagnosis, $n$} \\
\hline Pulmonary Infection, RR & 15 \\
\hline Pulmonary edema, $\mathrm{CHF}$ & 5 \\
\hline Complicated surgery & 4 \\
\hline $\mathrm{SAH} / \mathrm{ICH}$ & 2 \\
\hline Abdominal sepsis & 1 \\
\hline Trauma, head injury & 1 \\
\hline \multicolumn{2}{|l|}{ Type, $n$} \\
\hline Medical & 24 \\
\hline Surgical & 4 \\
\hline APACHE II & $22,2 \pm 0,9$ \\
\hline Predicted death rate, $\%$ & 43,1 \\
\hline SOFA & $10,1 \pm 0,6$ \\
\hline Outcome (mortality \%) & $10 / 28(35,7)$ \\
\hline MV duration, days & $24,5 \pm 5,1$ \\
\hline ICU LOS (median), days & $31,6 \pm 5,7(21)$ \\
\hline Fever, $>38.2^{\circ} \mathrm{C}$ & $12 / 28$ \\
\hline Low-dose steroids, $n$ & $17 / 28$ \\
\hline Shock, nor-adrenaline, $n$ & $27 / 28$ \\
\hline LMWH, $n$ & $17 / 28$ \\
\hline Fondaparinux (Arixtra), $n$ & $9 / 28$ \\
\hline Transfusions, $n$, (mean) & $18 / 28(2,9 \pm 0,7$ units $)$ \\
\hline Phenytoin (Epanutin), $n$ & $3 / 28$ \\
\hline Albumin, g/dL & $3,1 \pm 0,1(3,7-5,2)$ \\
\hline Corrected calcium, mg/dL & $8,2 \pm 0,3(8,5-10,5)$ \\
\hline Phosphorus, mg/dL & $3,4 \pm 0,2(2,5-4,8)$ \\
\hline Alkaline phosphatase, U/L & $120,0 \pm 1,4(42-141)$ \\
\hline Urea, mg/dL & $53,9 \pm 5,3(10-50)$ \\
\hline AST (SGOT), IU/dL & $44,4 \pm 9,8(9-36)$ \\
\hline ALT (SGPT), IU/dL & $68,9 \pm 28,9(20-28)$ \\
\hline $\mathrm{WBC}, / \mathrm{mm}^{3}$ & $11,087 \pm 1,415$ \\
\hline Creatinine, $\mathrm{mg} / \mathrm{dL}$ & $1,1 \pm 0.7(0,6-1,4)$ \\
\hline CRP, mg/dL & $108,4 \pm 20,1(<3,16)$ \\
\hline \multicolumn{2}{|l|}{ Food supplementation, $n$} \\
\hline Enteral & $28 / 28$ \\
\hline Parenteral & 2 \\
\hline Both & $2 / 28$ \\
\hline Micronutrients & $28 / 28$ \\
\hline Vitamins & $28 / 28$ \\
\hline
\end{tabular}

In parenthesis data represents range, $n$, percentages, or normal values. RR: respiratory failure, ICH: intracerebral hemorrhage, SAH: subarachnoid hemorrhage, CHF: congestive heart failure, LMWH: low molecular weight heparin, CRP: c-reactive protein, ICU: intensive care medicine, WBC: white blood cell, APACHE II: Acute Physiology And Chronic Health Evaluation, SOFA: Sequential Organ Failure Assessment, AST: Aspartate Aminotransferase, and ALT: Alanine Aminotransferase.

controls. There was a statistically significant difference in $\beta$ CTX levels between controls and baseline, 1st, 2nd, and 3rd week (Figure 1(a)).
3.2.3. Bone Formation. Serum PINP (Table 2, Figure 1(b)) were normal in $44.8 \%$ of the patients on admission (baseline) and below normal in the rest of the patients. On the 1st week serum levels were normal in 50\%, were below normal in $39.3 \%$, and above normal in $10.7 \%$ of the patients. On the 2nd week serum levels were normal in $63.2 \%$, were below normal in $10.5 \%$, and above normal in $26.3 \%$ of the patients. On the 3rd, 4th, and 5th week serum levels were normal in $60 \%, 37.5 \%$, and $28.6 \%$ and above normal in $40 \%, 62.5 \%$, and $71.4 \%$ of the patients, respectively. There was a statistically significant difference between controls and baseline and 1st week; between baseline and 2nd, 3rd, 4th, and 5th week; and between 1st and 3rd, 4th, and 5th week. Serum levels were increased by 16 -fold on 4 th and 22 -fold on 5 th week compared to baseline values. On 4th week and afterwards none of the patients had below normal levels.

3.2.4. Parathyroid Hormone and 25-Hydroxyvitamin D. All patients had decreased $25-\mathrm{OH}$ vitamin D serum levels on ICU admission compared to controls and were not restored to normal levels during the whole ICU stay (Figure 1(c)). Controls had significantly higher levels compared to the patients at all time-points. In parallel hypocalcaemia was observed at baseline (Table 1).

At baseline, $86.2 \%$ of the patients had increased serum iPTH levels which were 4 -fold times greater than controls. On 2nd, 3rd, and 4 th week $57.9 \%, 50 \%$, and $28.5 \%$ of the patients still had increased iPTH values (Figure 1(d)). There was a statistically significant difference between controls and baseline and 1st week and between baseline and 5th week serum levels. We did not find any correlation between severity of illness (APACHE II) and iPTH or 25-OH vitamin D levels. Also, no correlation was found among iPTH and CTX values on admission, nor among iPTH and illness severity, ICU LOS, and SOFA scores.

3.2.5. Measurements according to Survival. We also performed analysis according to outcomes (Table 3 and Figures 2 and 3). Survivors had significantly shorter duration of mechanical ventilation (MV) $(p=0.039)$ and duration of ICU stay $(p=0.01)$ from nonsurvivors. Both survivors and especially nonsurvivors $25-\mathrm{OH}$ vitamin $\mathrm{D}$ serum levels were significantly lower compared to normal throughout the whole ICU stay. iPTH serum levels were in both groups higher on admission tending to normalization on 4 th to 5 th week of ICU stay. $\beta$-CTx serum levels were higher compared to controls on admission and increased until the 2 nd week of ICU stay in both survivors and nonsurvivors. On 3 rd week and thereafter $\beta$-CTx serum levels decreased in both groups but were still higher compared to controls. PINP serum levels were lower compared to controls in both groups on admission but were increased overcoming control levels on 4th and 5th week of ICU stay in both groups.

\section{Discussion}

The major findings of this study are (1) critically ill patients had very low serum levels of vitamin D during ICU stay, 
TABLE 2: Values for BTMs obtained from controls versus patients over time (weeks) of ICU stay.

\begin{tabular}{|c|c|c|c|c|c|c|c|}
\hline Parameter & 4) & 8) & 8) & ) & 1) & $4 \mathrm{t}$ & 5 th $(n=7)$ \\
\hline & 0.4 & 9) & 0) & 5) & 1,2 & 0,96 & $\begin{array}{c}1.15 \\
(0,79-1,30)\end{array}$ \\
\hline $1 \mathrm{~N}$ & & 12,7 & 20 , & & & & $\begin{array}{c}281,1 \\
(56,8-460,7)\end{array}$ \\
\hline iPTH & & 175,4 & 102,5 & 88,4 & 96,5 & 63,7 & $\begin{array}{c}66,1 \\
(20,2-71,3)\end{array}$ \\
\hline 25-OH VitD* & $24.1(22.9-27.1)$ & $3,4(2,0-6,1)$ & $4,9(2,0-9,8)$ & $4,9(2,0-9,1)$ & $3,8(2,0-8,1)$ & $6,6(3,6-8,4)$ & $5,1(2,0-6,8)$ \\
\hline
\end{tabular}

${ }^{*} \mathrm{ng} / \mathrm{mL}$ values are expressed as median $( \pm \mathrm{IQR})$.

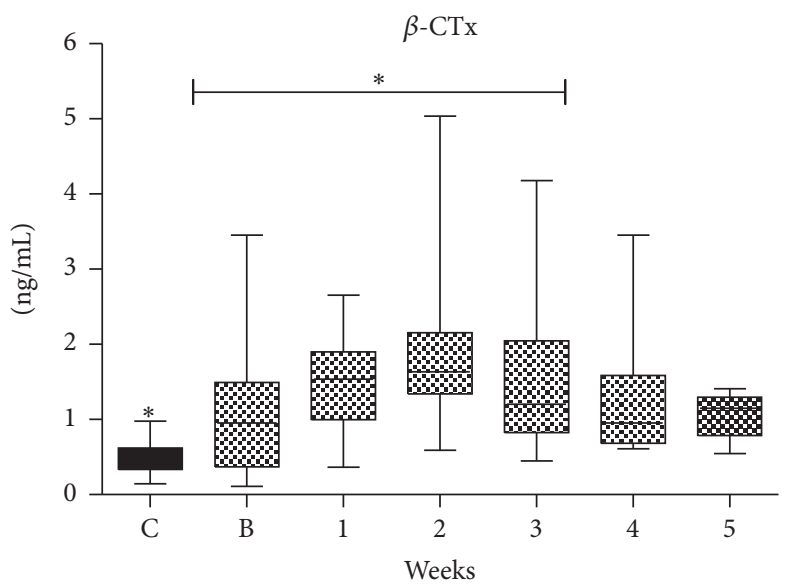

(a)

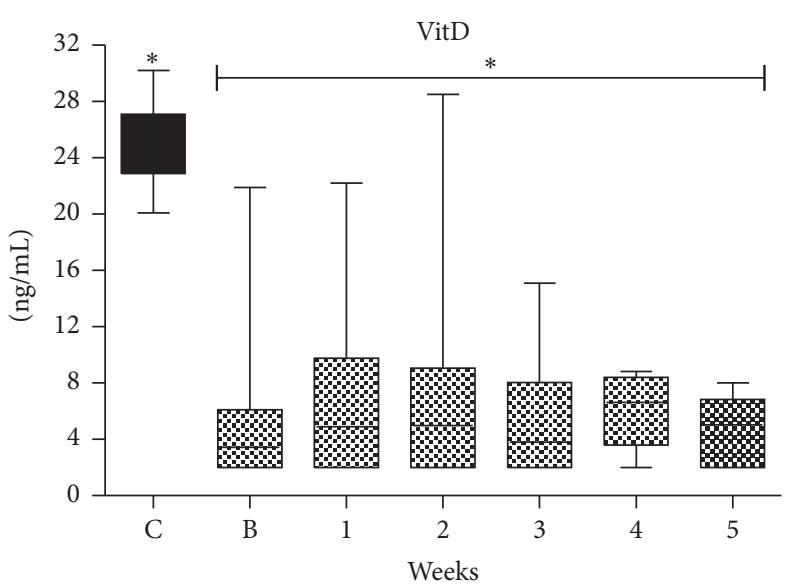

(c)

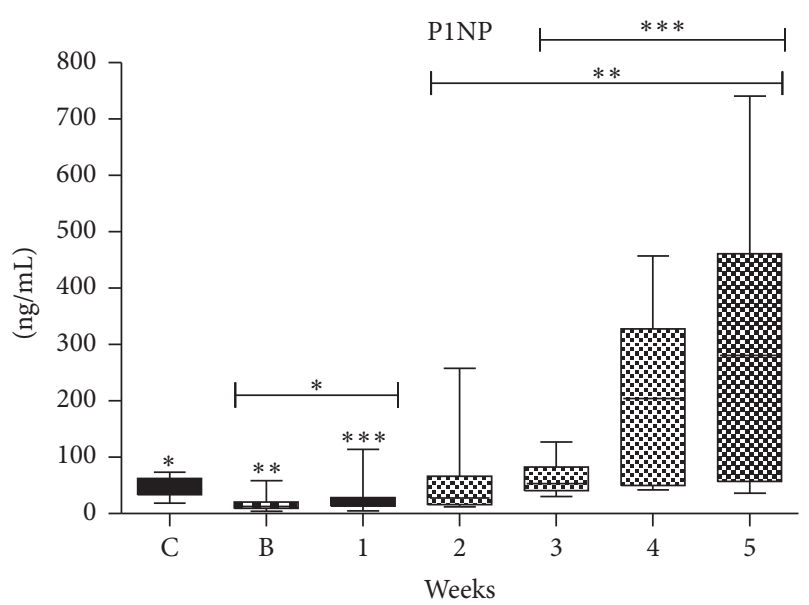

(b)

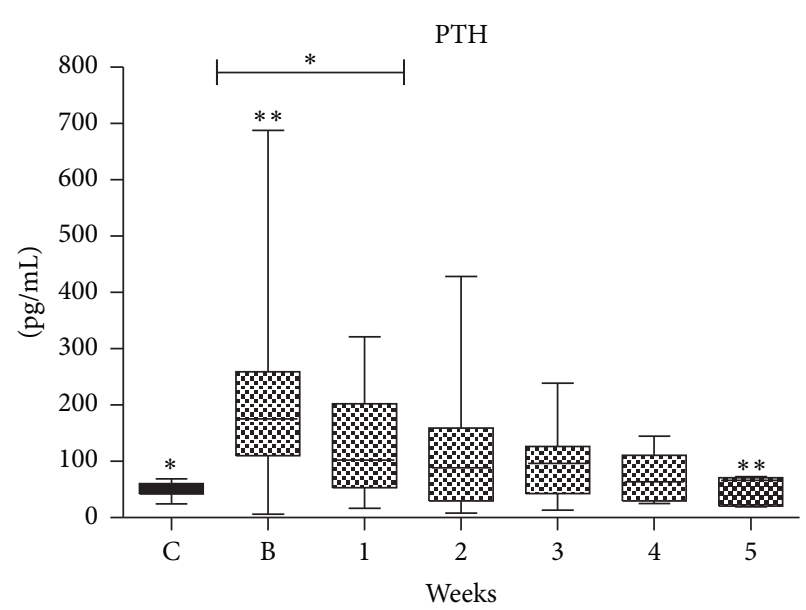

(d)

FIGURE 1: BTMs course during ICU stay in whole population. (a) CTx; (b) PINP; (c) Vitamin D; (d) Parathormone. Values are presented as media $( \pm \mathrm{IQR}) . \mathrm{C}=$ controls; $\mathrm{B}=$ baseline; numbers: weeks. Symbols $*, * *$, and $* * *$ indicate statistically significant difference $(p<0.05)$.

whereas increased iPTH levels on admission were normalized in the majority of patients by the 4 th week. (2) $\beta$-CTx serum levels were increased during ICU stay with a peak on 2 nd week. (3) PINP serum levels were lower compared to controls on admission but increased significantly on the 4 th to 5 th week (5-fold) of follow-up.

Overall we observed a change of BTMs from increased bone resorption especially through the first 2 weeks of ICU stay, to increased bone formation after the 3rd week.
Our findings suggest a biphasic model of BTMs changes that is of extensive initial bone resorption pattern which is then partially counterbalanced by bone formation. Our findings are also indicative of altered bone turnover in a selected population of critically ill patients which is consistent with current literature showing an association between the duration of critical illness ( $>5$ days) and bone resorption $[1,10]$. This BTMs pattern is consistent with previous studies that reported increased bone resorption markers [4, 8-14], 
TABLE 3: Demographics, admission diagnosis, and characteristics of the patients according to survival status $(n=28)$.

\begin{tabular}{|c|c|c|c|}
\hline Variable & Survivors $(n=18)$ & Nonsurvivors $(n=10)$ & $p$ \\
\hline Age, (range) yrs & $65.0 \pm 2.5$ & $71.6 \pm 3.2$ & 0.88 \\
\hline Males, $n, \%$ & $10 / 18,55.5 \%$ & $6 / 10,60.0 \%$ & 1.0 \\
\hline \multicolumn{4}{|l|}{ Admission diagnosis, $n$} \\
\hline Pulmonary Infection, RR & 10 & 5 & \\
\hline Pulmonary edema, CHF & 3 & 2 & \\
\hline Complicated surgery & 2 & 2 & \\
\hline $\mathrm{SAH} / \mathrm{ICH}$ & 1 & 1 & \\
\hline Abdominal sepsis & 1 & & \\
\hline Trauma, head injury & 1 & & \\
\hline \multicolumn{4}{|l|}{ Type, $n$} \\
\hline Medical & 16 & 8 & \\
\hline Surgical & 2 & 2 & \\
\hline APACHE II & $21.9 \pm 1.2$ & $22.7 \pm 1.5$ & 0.58 \\
\hline SAPS II & $62.9 \pm 2.5$ & $60.1 \pm 3.5$ & 0.83 \\
\hline SOFA & $9.9 \pm 0.9$ & $10.5 \pm 0.9$ & 0.65 \\
\hline MV duration, days & $14.3 \pm 2.8$ & $42.8 \pm 11.7$ & 0.039 \\
\hline ICU LOS (median), d & $18.6 \pm 2.9(13)$ & $55.0 \pm 12.1(51.5)$ & 0.01 \\
\hline Fever, $>38.2^{\circ} \mathrm{C}$ & $8 / 18,44,4 \%$ & $4 / 10,40 \%$ & 1.0 \\
\hline Corrected calcium, mg/dL & $7.9 \pm 0.2$ & $8.5 \pm 0.6$ & 0.65 \\
\hline Phosphorus, mg/dL & $3.2 \pm 0.3$ & $3.8 \pm 0.3$ & 0.13 \\
\hline Alkaline phosphatase, $\mathrm{mg} / \mathrm{dL}$ & $117.7 \pm 19.5$ & $124.0 \pm 17.1$ & 0.18 \\
\hline Urea, mg/dL & $54.5 \pm 7.1$ & $52.8 \pm 8.3$ & 0.96 \\
\hline AST (SGOT), IU/dL & $51.4 \pm 14.9$ & $31.8 \pm 4.6$ & 1.0 \\
\hline ALT (SGPT), IU/dL & $90.1 \pm 44.4$ & $31.1 \pm 5.9$ & 0.98 \\
\hline $\mathrm{WBC}, / \mathrm{mm}^{3}$ & $10,964 \pm 1,997$ & $11,309 \pm 1,804$ & 0.79 \\
\hline Creatinine, $\mathrm{mg} / \mathrm{dL}$ & $1.1 \pm 0.8$ & $1.2 \pm 0.2$ & 0.51 \\
\hline Albumin, $\mathrm{g} / \mathrm{dL}$ & $3.0 \pm 0.1$ & $3.3 \pm 0.2$ & 0.38 \\
\hline CRP, mg/dL & $112.1 \pm 24.2$ & $97.8 \pm 38.9$ & 0.75 \\
\hline i-CTx (ng/mL) & $0,96 \pm 0,19$ & $1,49 \pm 0,32$ & 0.098 \\
\hline P1NP (ng/mL) & $15,46 \pm 2,44$ & $19,85 \pm 5,34$ & 0.773 \\
\hline i-PTH (pg/mL) & $215,9 \pm 39,68$ & $159,6 \pm 32,68$ & 0.533 \\
\hline 25-OH Vit. D (ng/mL) & $6,45 \pm 1,49$ & $3,80 \pm 0,94$ & 0.433 \\
\hline Low-dose steroids, $n$ & $12 / 18$ & $4 / 10$ & \\
\hline Shock, nor-adrenaline, $n$ & $17 / 18$ & $10 / 10$ & \\
\hline LMWH, $n$ & $14 / 18$ & $5 / 10$ & \\
\hline Fondaparinux (Arixtra), $n$ & $4 / 18$ & $5 / 10$ & \\
\hline Transfusions, $n$, (mean) & $10 / 18$ & $8 / 10$ & \\
\hline Phenytoin (Epanutin), $n$ & $2 / 18$ & $1 / 10$ & \\
\hline Food supplementation, $n$ & $18 / 18$ & $10 / 10$ & \\
\hline Enteral & $18 / 18$ & $10 / 10$ & \\
\hline Parenteral & $1 / 18$ & $1 / 10$ & \\
\hline Both & $1 / 18$ & $1 / 10$ & \\
\hline Micronutrients & $18 / 18$ & $10 / 10$ & \\
\hline Vitamins & $18 / 18$ & $10 / 10$ & \\
\hline
\end{tabular}

RR: respiratory failure, ICH: intracerebral hemorrhage, SAH: subarachnoid hemorrhage, CHF: congestive heart failure, LMWH: low molecular weight heparin, CRP: c-reactive protein, ICU: intensive care medicine, WBC: white blood cell, APACHE II: Acute Physiology And Chronic Health Evaluation, SOFA: Sequential Organ Failure Assessment, AST: Aspartate Aminotransferase, and ALT: Alanine Aminotransferase. 


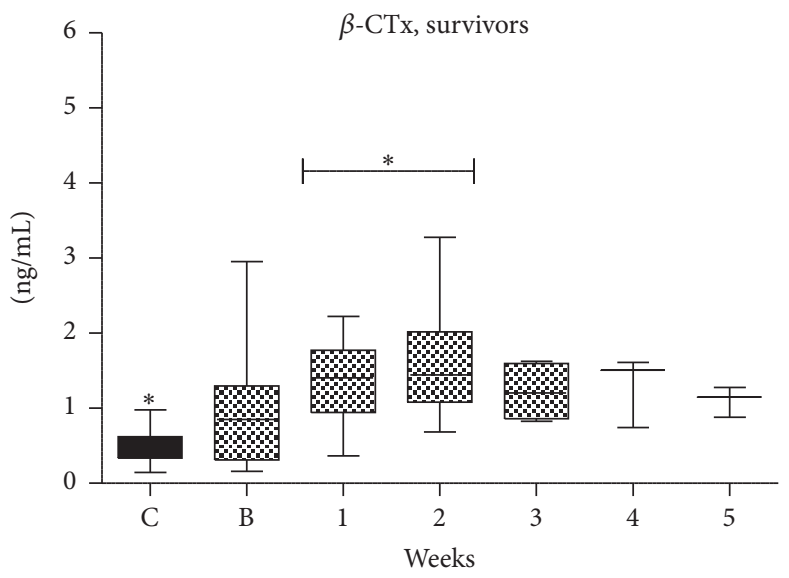

(a)

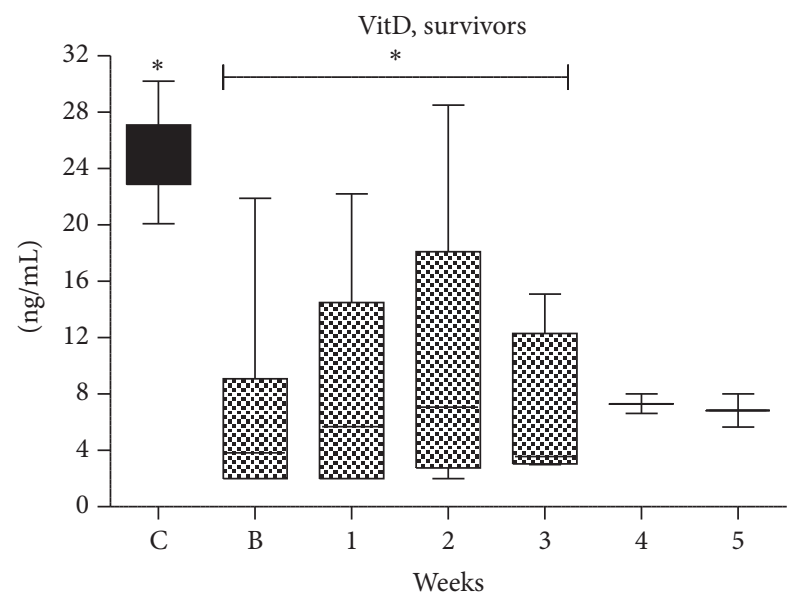

(c)

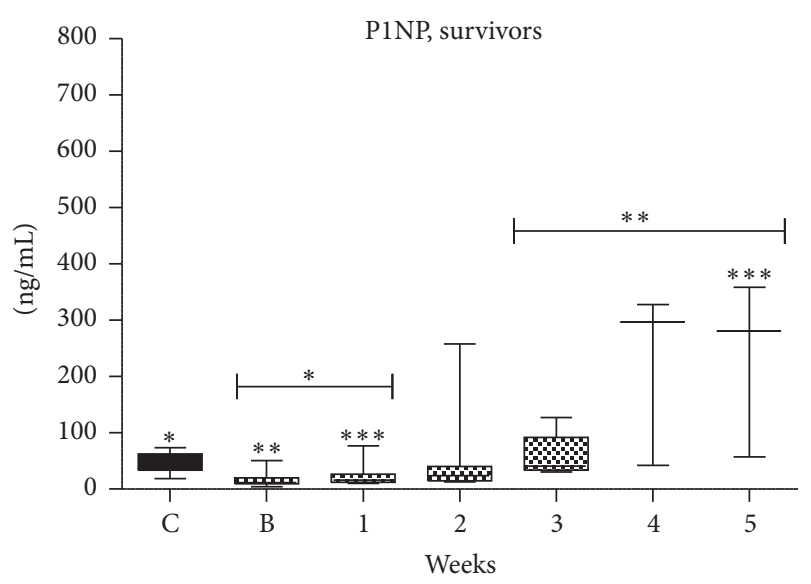

(b)

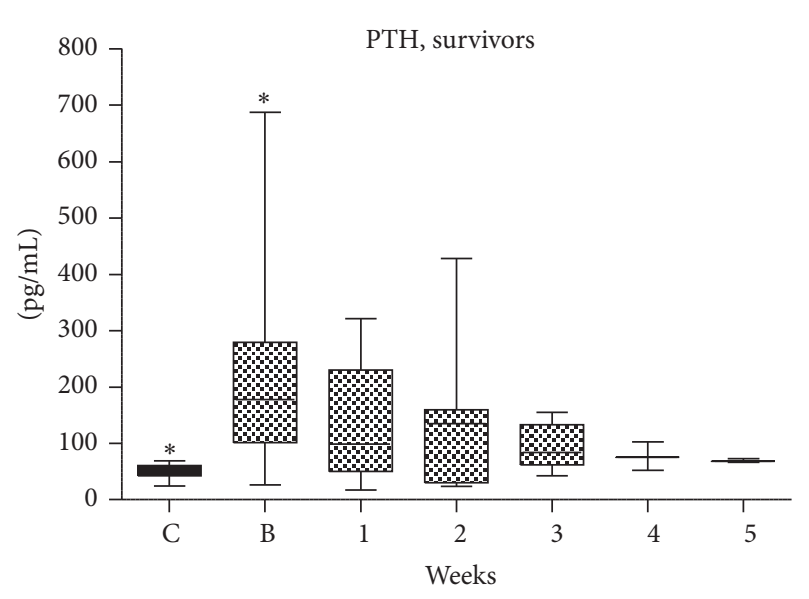

(d)

Figure 2: BTMs course during ICU stay in survivors. (a) CTx; (b) PINP; (c) Vitamin D; (d) Parathormone. Values are presented as media $( \pm \mathrm{IQR}) . \mathrm{C}=$ controls; $\mathrm{B}=$ baseline; numbers: weeks. Symbols $*, * *$, and $* * *$ indicate statistically significant difference $(p<0.05)$.

increased serum osteoclast precursors [15], increased bone formation, and decreased osteocalcin during critical illness compared with controls $[4,11,16]$. These studies consistently described changes: an increased osteoclastic bone resorption (increased urinary $\mathrm{DpD}$ and $\mathrm{PyD}$, serum B-CTX/ICTP), an increase in immature osteoblast number and activity (serum $\mathrm{P} 1 \mathrm{CP}$ and PINP), and a reduced activity of mature osteoblasts (serum OC and ALP) [4, 8, 10-17].

Our biphasic model of BTMs changes cannot be generalized in every ICU patient and may be partially explained due to longer follow-up period (median 21 days) compared to previous studies in which the follow-up period was up to 10 days $[4,8,10-17]$. We also had baseline and weekly BTMs measurements and not single measurement on admission or discharge as previous studies [1].

We measured serum PINP and $\beta$-CTX levels as the most promising BTMs by the Joint International Bone Markers Standards Working Group [7] which however were occasionally evaluated and especially $\beta$-CTX in two studies and PINP in one study. Consistently to our results $\beta$-CTX was increased by 3 - to 6 -fold compared to controls or reference values in two studies [4, 8]. Also, PINP was increased by 1.7 -fold on
ICU discharge [4]. However these studies monitored patients for up to 10 days and measured BMT only once without evaluating calcium, iPTH, or vitamin D.

A very recent study found increased CTx and decreased serum PINP levels during ICU stay which is in accordance with our findings [9]. In addition to this study we found a commensurate response in bone formation markers during critical illness, that is, after the 3rd week of ICU stay as stated above. However, we have significant methodological differences compared to this study because we enrolled both survivors and not nonsurvivors, patients with longer duration of MV and ICU LOS, and multiple time-points of BTMs measurements during ICU stay.

In accordance with our study patients had during their ICU stay insufficient vitamin D serum levels, increased iPTH serum levels, and hypocalcaemia [9]. It was also suggested that vitamin D deficiency in critically ill patients with resultant secondary hyperparathyroidism along with prolonged immobilization and other metabolic abnormalities may increase the risk of excessive bone resorption $[1,17]$. However, correction of vitamin D deficiency in critically ill patients did not normalize bone resorption markers, 


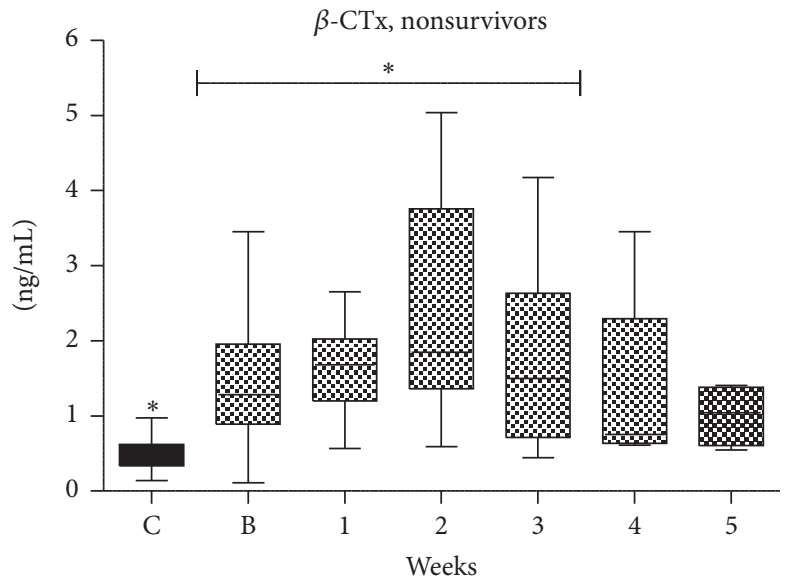

(a)

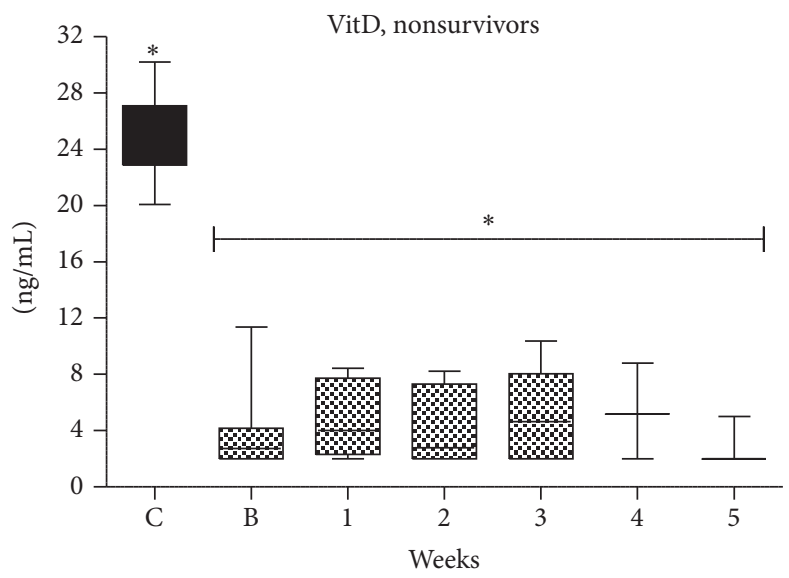

(c)

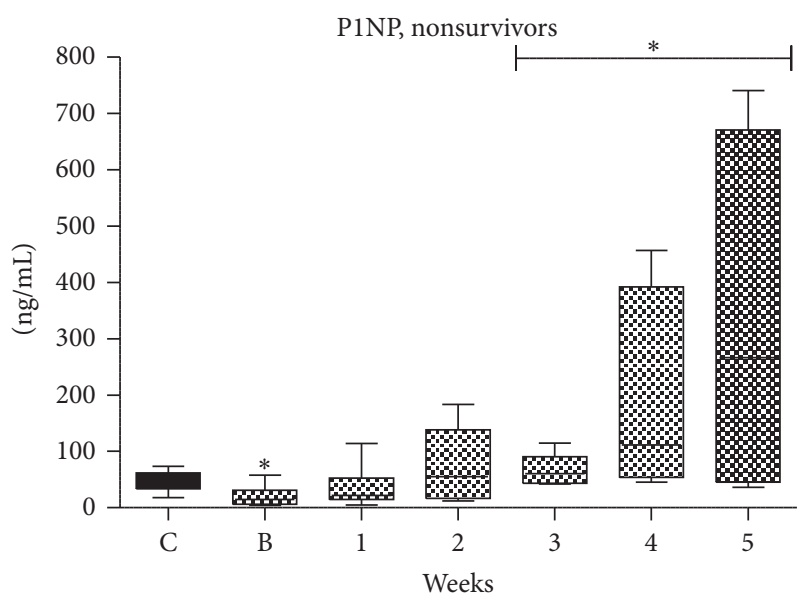

(b)

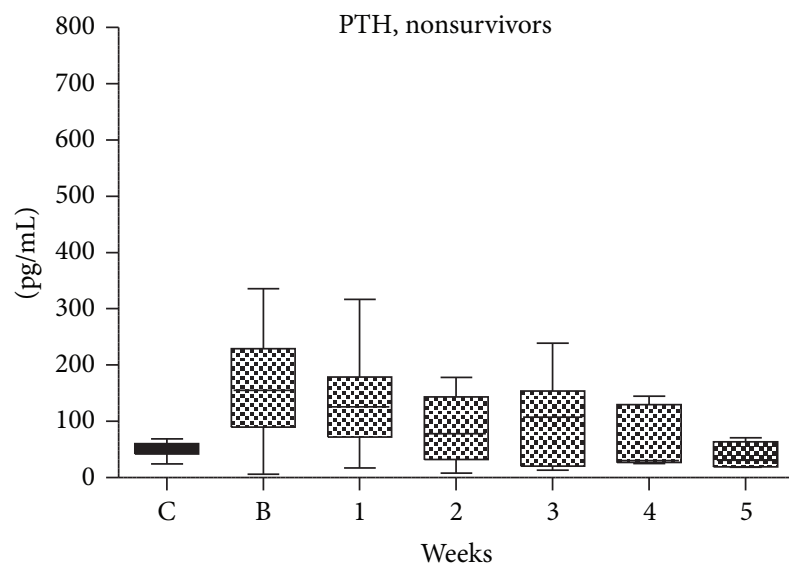

(d)

FIgURE 3: BTMs course during ICU stay in nonsurvivors. (a) CTx; (b) PINP; (c) Vitamin D; (d) Parathormone. Values are presented as media $( \pm \mathrm{IQR}) . \mathrm{C}=$ controls; $\mathrm{B}=$ baseline; numbers: weeks. Symbols $*, * *$, and $* * *$ indicate statistically significant difference $(p<0.05)$.

suggesting vitamin D deficiency is not the only mechanism for accelerated bone turnover [4, 12]. Hypovitaminosis D is common and multifactorial in critically ill patients but several pathophysiological and iatrogenic interventions and particularly during acute hemodilution have impact on 25$\mathrm{OH}-\mathrm{D}$ assay accuracy and results, making the assessment of vitamin $\mathrm{D}$ status during critical care hazardous. Also, the best option to measure 25-OH-D blood concentration has not been definitely defined [18].

4.1. Limitations of the Study. An important limitation of our study is that we do not report changes in bone mineral density (BMD) during or following critical illness. However, measurements of BTMs on ICU discharge may be a useful tool to identify patients requiring BMD measurement after ICU. Also, the relatively small number of patients, the short duration of follow-up, the absence of post-ICU follow-up, and a lack of premorbid assessment of BTMs or skeletal health may be disadvantages of the study. The short duration of follow-up decreases the ability to establish a causal relationship between critical illness and bone turnover [1].
Another important limitation of the study is the lack of analysis of the effect of possible confounding variables on the association between critical illness and altered bone turnover. The effects of other known causes of osteoporosis and variables, known to affect the metabolism of BTMs (including sex, menopausal status, renal failure, liver disease, diabetes, thyroid disease, inflammation, and medications and particularly glucocorticoids), were not consistently addressed. Also, the use of low dose hydrocortisone as part of sepsis management in most of the patients $(\approx 61 \%)$ could affect markers levels. Also, infections like pneumonia may affect PINP levels in the circulation [19]. Finally the study was not designed to identify differences among survivors and nonsurvivors and thus further analysis could not be performed.

\section{Conclusions}

In this prospective observational study we found that critically ill patients receiving $\mathrm{MV}$ had a pattern of hypovitaminosis D, increased iPTH, hypocalcaemia, and a BTM compatible with altered bone metabolism and accelerated 
bone turnover consistent with increased bone loss and only partial commensurate response regarding bone formation. This model of biphasic BTMs changes in critically ill patients during ICU stay is described for first time. Our findings suggest that further studies are needed to confirm our data incorporating BMD changes during and after critical illness, identifying risk factors and the extent of critical illness related to bone turnover changes. Finally, more studies are needed to design appropriate preventive measures including physical therapy and therapeutic strategies.

\section{Abbreviations}

$\begin{array}{ll}\text { APACHE II score: } & \text { Acute Physiologic Assessment and } \\ & \text { Chronic Health Evaluation } \\ \text { BMD: } & \text { Bone mineral density } \\ \text { BTMs: } & \text { Bone turnover markers } \\ \beta \text {-CTx: } & \begin{array}{l}\text { C-telopeptide fragments of } \\ \text { collagen type I al chains }\end{array} \\ \text { ICU: } & \text { Intensive care unit } \\ \text { iPTH: } & \text { Intact parathormone } \\ \text { LOS: } & \text { Length of stay } \\ \text { LMWH: } & \text { Low molecular weight heparin } \\ \text { MV: } & \text { Mechanical ventilation } \\ \text { PINP: } & \text { N-terminal propeptide of type I } \\ & \text { procollagen } \\ \text { 25-OH vitamin } & \text { D: } \\ \text { SOFA score: } & \text { Sepsis-Related Organ Failure } \\ & \text { Assessment. }\end{array}$

\section{Competing Interests}

The authors declare that they have no conflict of interests.

\section{Authors' Contributions}

Alexandra Gavala and Anna Korompeli participated in data collection and preparation of the manuscript. Konstantinos Makris participated in study design, biochemistry measurements, and preparation of the manuscript, AND Pavlos Myrianthefs participated in study design, collection of the data, and preparation of the manuscript.

\section{Acknowledgments}

The study was partially funded from the Special Account for Research Grants of the National and Kapodistrian University of Athens.

\section{References}

[1] N. Orford, C. Cattigan, S. L. Brennan, M. Kotowicz, J. Pasco, and D. J. Cooper, "The association between critical illness and changes in bone turnover in adults: a systematic review," Osteoporosis International, vol. 25, no. 10, pp. 2335-2346, 2014.

[2] D. M. Griffith and T. S. Walsh, "Bone loss during critical illness: a skeleton in the closet for the intensive care unit survivor?" Critical Care Medicine, vol. 39, no. 6, pp. 1554-1556, 2011.
[3] M. A. Via, E. J. Gallagher, and J. I. Mechanick, "Bone physiology and therapeutics in chronic critical illness," Annals of the New York Academy of Sciences, vol. 1211, pp. 85-94, 2010.

[4] G. Van Den Berghe, D. Van Roosbroeck, P. Vanhove, P. J. Wouters, L. De Pourcq, and R. Bouillon, "Bone turnover in prolonged critical illness: effect of vitamin D," Journal of Clinical Endocrinology and Metabolism, vol. 88, no. 10, pp. 4623-4632, 2003.

[5] M. A. Via, E. J. Gallagher, and J. I. Mechanick, "Bone physiology and therapeutics in chronic critical illness," Annals of the New York Academy of Sciences, vol. 1211, pp. 85-94, 2010.

[6] N. R. Orford, K. Saunders, E. Merriman et al., "Skeletal morbidity among survivors of critical illness," Critical Care Medicine, vol. 39, no. 6, pp. 1295-1300, 2011.

[7] S. Vasikaran, R. Eastell, O. Bruyère et al., "Markers of bone turnover for the prediction of fracture risk and monitoring of osteoporosis treatment: a need for international reference standards," Osteoporosis International, vol. 22, no. 2, pp. 391420, 2011.

[8] L. Lind, F. Carlstedt, J. Rastad et al., "Hypocalcemia and parathyroid hormone secretion in critically ill patients," Critical Care Medicine, vol. 28, no. 1, pp. 93-99, 2000.

[9] N. R. Orford, S. E. Lane, M. Bailey et al., "Changes in bone mineral density in the year after critical illness," American Journal of Respiratory and Critical Care Medicine, vol. 193, no. 7, pp. 736-744, 2016.

[10] S. A. Shapses, C. Weissman, M. J. Seibel, and H. A. Chowdhury, "Urinary pyridinium cross-link excretion is increased in critically ill surgical patients," Critical Care Medicine, vol. 25, no. 1, pp. 85-90, 1997.

[11] G. Van den Berghe, R. C. Baxter, F. Weekers et al., "The combined administration of GH-releasing peptide-2 (GHRP2 ), TRH and GnRH to men with prolonged critical illness evokes superior endocrine and metabolic effects compared to treatment with GHRP-2 alone," Clinical Endocrinology, vol. 56, no. 5, pp. 655-669, 2002.

[12] D. M. Nierman and J. I. Mechanick, "Biochemical response to treatment of bone hyperresorption in chronically critically ill patients," Chest, vol. 118, no. 3, pp. 761-766, 2000.

[13] G. Van Den Berghe, F. Weekers, R. C. Baxter et al., "Fiveday pulsatile gonadotropin-releasing hormone administration unveils combined hypothalamic-pituitary-gonadal defects underlying profound hypoandrogenism in men with prolonged critical illness," Journal of Clinical Endocrinology and Metabolism, vol. 86, no. 7, pp. 3217-3226, 2001.

[14] L. M. Smith, B. Cuthbertson, J. Harvie, N. Webster, S. Robins, and S. H. Ralston, "Increased bone resorption in the critically ill: association with sepsis and increased nitric oxide production," Critical Care Medicine, vol. 30, no. 4, pp. 837-840, 2002.

[15] H. C. Owen, I. Vanhees, L. Solie et al., "Critical illnessrelated bone loss is associated with osteoclastic and angiogenic abnormalities," Journal of Bone and Mineral Research, vol. 27, no. 7, pp. 1541-1552, 2012.

[16] G. Van den Berghe, P. Wouters, F. Weekers et al., "Reactivation of pituitary hormone release and metabolic improvement by infusion of growth hormone-releasing peptide and thyrotropinreleasing hormone in patients with protracted critical illness," The Journal of Clinical Endocrinology \& Metabolism, vol. 84, no. 4, pp. 1311-1323, 1999.

[17] D. M. Nierman and J. I. Mechanick, "Bone hyperresorption is prevalent in chronically critically ill patients," Chest, vol. 114, no. 4, pp. 1122-1128, 1998. 
[18] A.-F. Rousseau, P. Damas, M. Janssens et al., "Critical care and vitamin D status assessment: what about immunoassays and calculated free 25OH-D?" Clinica Chimica Acta, vol. 437, pp. 4347, 2014.

[19] C. Nordenbæk, B. Teisner, and P. Junker, "Connective tissue responses in acute community-acquired pneumonia," Respiratory Medicine, vol. 97, no. 6, pp. 660-666, 2003. 


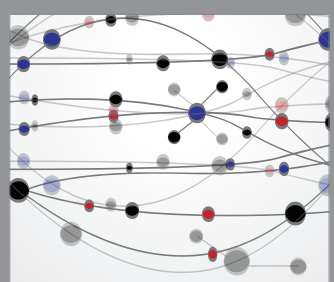

The Scientific World Journal
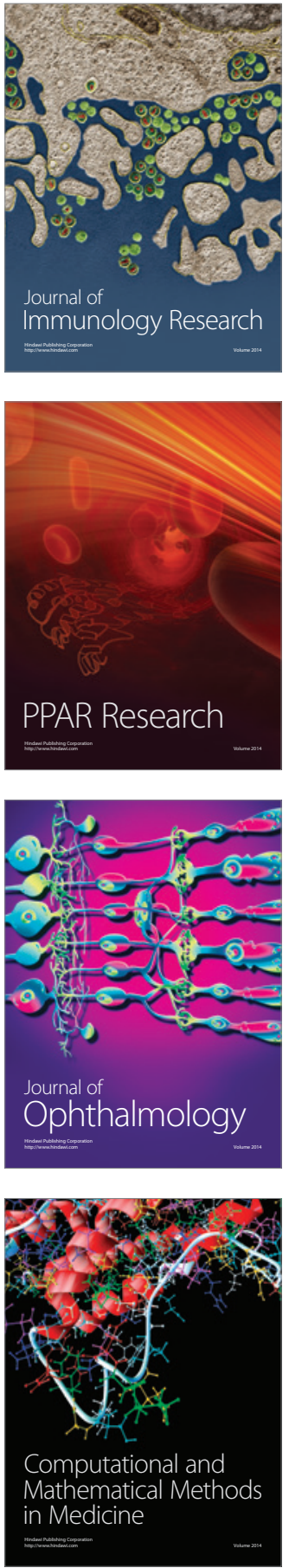

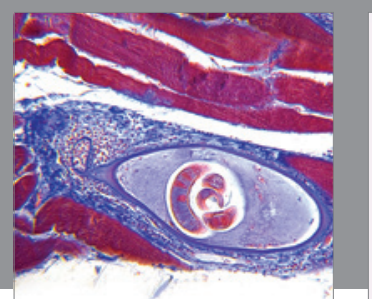

Gastroenterology Research and Practice

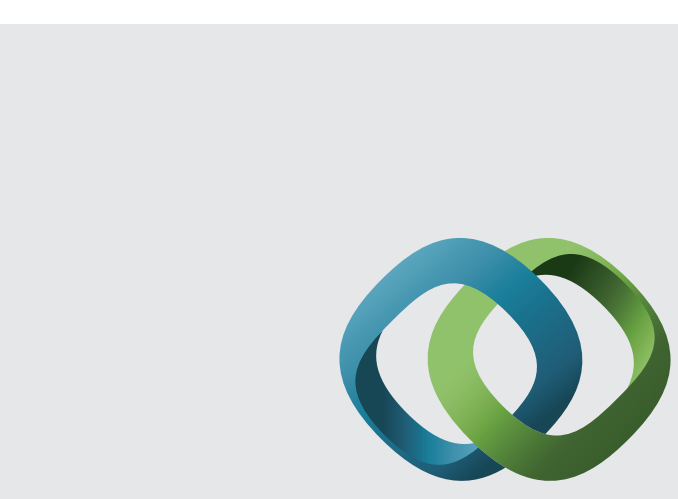

\section{Hindawi}

Submit your manuscripts at

http://www.hindawi.com
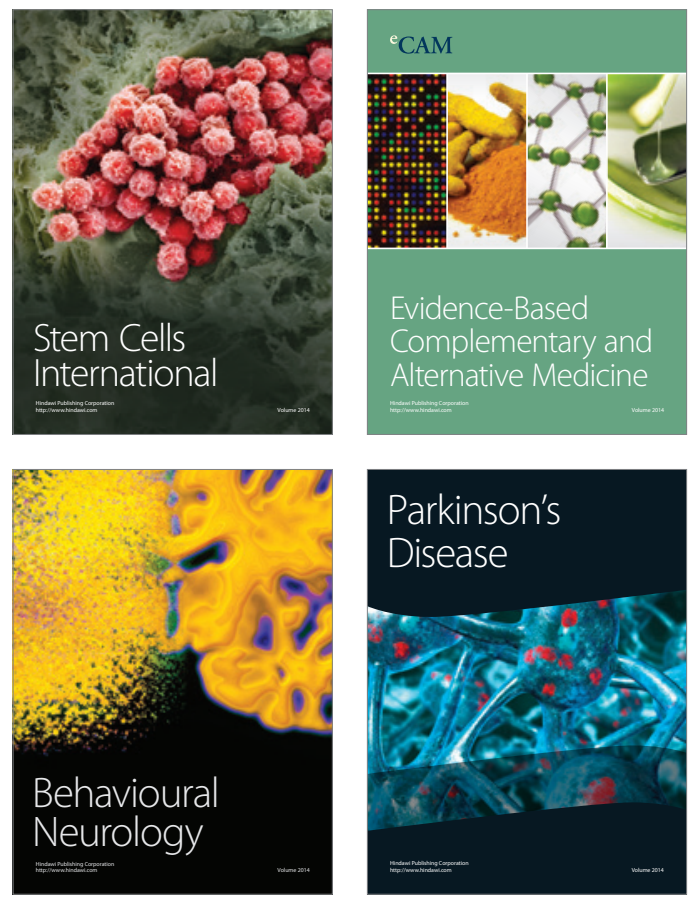
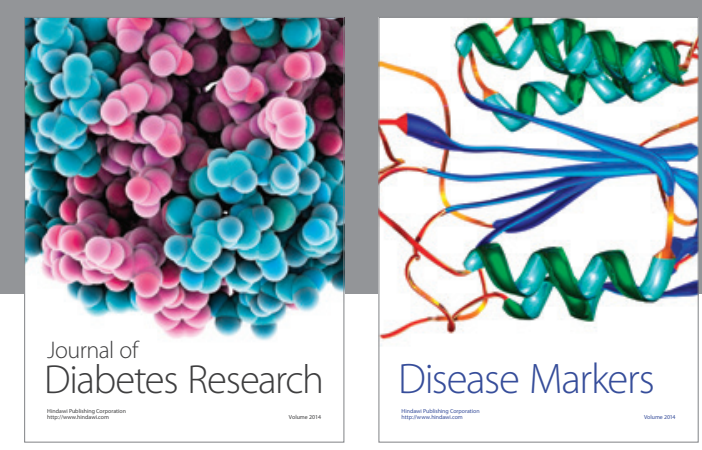

Disease Markers
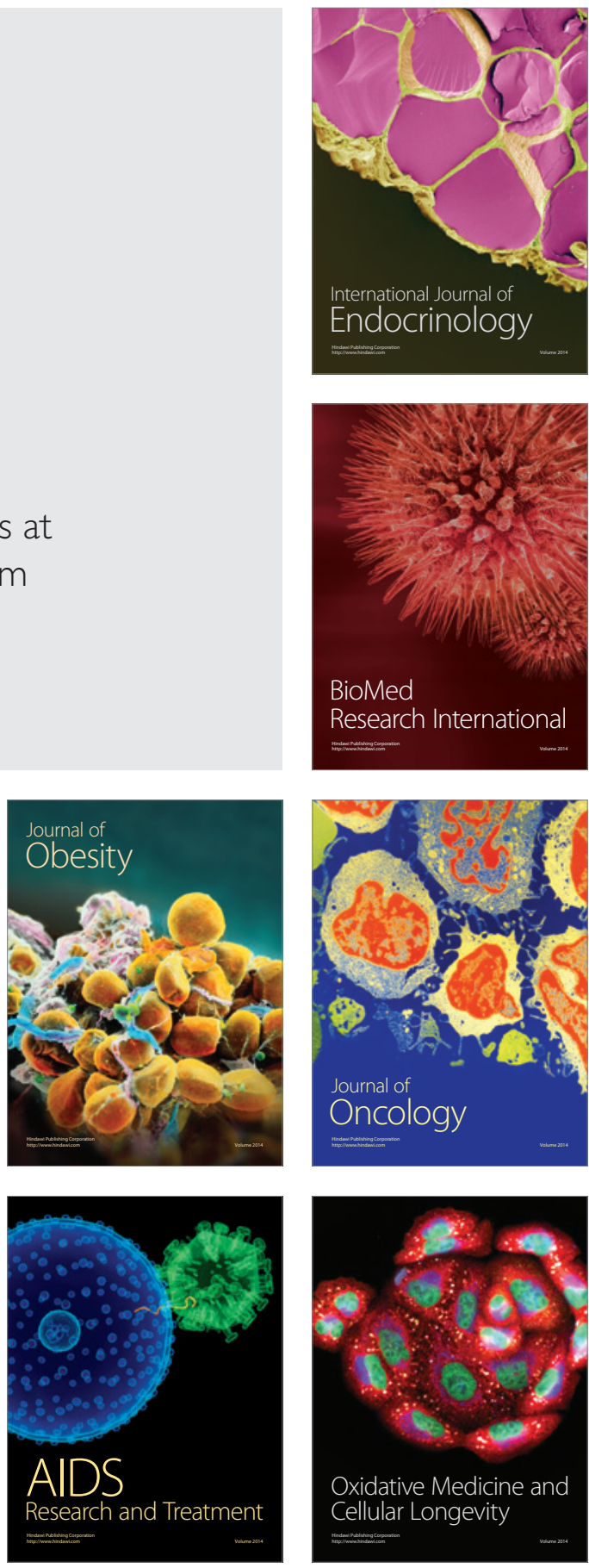